\title{
Leucemias neonatales: experiencia en el Hospital Infantil de México Federico Gómez (HIMFG)
}

Ibarra-Ríos $D^{1}$, Zapata-Tarres $M^{2}$, Villanueva-García $D^{3}$, Peña-del Castillo $H^{4}$, Dorantes-Acosta $\mathrm{EM}^{5}$, Juárez-Villegas $\mathrm{LE}^{6}$

\section{Resumen}

INTRODUCCIÓN: la leucemia neonatal (o congénita) es aquella diagnosticada en los primeros 30 días tras el nacimiento. Su incidencia se reporta en 1 a 5 por cada millón de recién nacidos vivos (corresponde a menos del $1 \%$ de las leucemias en pediatría).

OBJETIVO: describir los casos diagnosticados en el Hospital Infantil de México Federico Gómez (HIMFG).

PRESENTACIÓN DE LA SERIE DE CASOS: se incluyó pacientes menores de 30 días a los cuales se les realizó el diagnóstico morfológico de leucemias entre el $1^{\circ}$ de enero de 1990 y el $1^{\circ}$ de junio del 2012 en el HIMFG.

RESULTADOS: en los 12 años del estudio se identificaron 3,061 casos de leucemias, de los cuales sólo cinco expedientes correspondieron a leucemias neonatales. Se describen tres pacientes femeninos y dos masculinos, con edad media al diagnóstico de 21 días (17-26 días), tratándose con mayor frecuencia de leucemia mieloide (LMA) en tres casos $(60 \%)$ y leucemia linfoide en dos pacientes. Un caso de LMA asociado a síndrome de Down. En ningún caso se encontraron translocaciones de riesgo. Cuatro pacientes $(80 \%)$ recibieron manejo para síndrome de lisis tumoral. Cuatro pacientes fallecieron con una mediana de edad de 84 días (57-176 días). El paciente que sobrevivió recibió tratamiento con doxorrubicina y metotrexate al $50 \%$ y en su último seguimiento a los tres años de edad continuaba en remisión.

CONCLUSIONES: la presentación de los casos corresponde a la reportada en literatura con respecto a frecuencias de leucemias linfoides y mieloides. La supervivencia global a largo plazo fue del $20 \%$ (33\% para las mieloides) con un solo paciente en remisión. Este padecimiento tiene un pronóstico fatal que puede mejorar con el diagnóstico oportuno (incluso prenatal) y manejo con quimioterapia intensiva. Ésta es la serie más grande de leucemias neonatales reportada en México.

PALABRAS CLAVE: leucemia neonatal, leucemia aguda linfoide, leucemia aguda mieloide serie de casos.
${ }^{1}$ Médico adscrito a la Unidad de Cuidados Neonatales, Hospital Infantil de México Federico Gómez. ${ }^{2}$ Médico adscrito al Servicio de Oncología, Instituto Nacional de Pediatría.

3Jefa de Servicio de Neonatología, Hospital Infantil de México Federico Gómez.

${ }^{4}$ Residente de Oncología Pediátrica, Dirección de Enseñanza, Instituto Nacional de Pediatría.

${ }^{5}$ Médico adscrito al Servicio de Oncología, Hospital Infantil de México Federico Gómez.

${ }^{6}$ Jefe del Departamento de Hemato-Oncología del Hospital Infantil de México Federico Gómez.

Recibido: 3 de noviembre del 2015

Aceptado: 17 de abril del 2017

Correspondencia

Dra. Marta Zapata-Tarres

mzapatatarres@gmail.com

Este artículo debe citarse como

Ibarra-Ríos D, Zapata-Tarres M, Villanueva-García D, Peña-del Castillo H, Dorantes-Acosta EM, JuárezVillegas LE. Leucemias neonatales: experiencia en el Hospital Infantil de México Federico Gómez (HIMFG). Acta Pediatr Mex. 2017;38(6):394-401. 


\section{Neonatal leukaemia: in the Hospital Infantil de México Federico Gómez. (HIMFG) Experience.} Ibarra-Ríos $D^{1}$, Zapata-Tarres $\mathrm{M}^{2}$, Villanueva-García $D^{3}$, Peña-del Castillo $\mathrm{H}^{4}$,
Dorantes-Acosta $\mathrm{EM}^{5}$, Juárez-Villegas $\mathrm{LE}^{6}$

\begin{abstract}
BACKROUND: Neonatal leukaemia is the one diagnosed in the first 30 days of life. Its incidence is reported in 1 to 5 per million of newborns (it represents less than $1 \%$ of leukaemias).

CASES PRESENTATION: We describe all cases diagnosed with neonatal leukaemia between January 1st 1990 and November 1, 2012 at our institution. We searched for all medical records labelled under the diagnosis of leukaemia, and we selected those in which the diagnosis was done in the first 30 days of life. A total of 3,061 medical records were labelled as leukaemia, from which only $5(0.16 \%)$ corresponded to neonatal leukaemia. Affected patients were three females and 2 males with a median age at diagnosis was 21 days (17-26 days). Three cases were acute myeloid leukaemia and two acute lymphoid leukaemia. One patient had Down syndrome. Four patients developed tumoral lysis syndrome. Four patients died at a median age of 84 days (57-176 days). The only survivor patient received complete $50 \%$ reduced ATEDox NOPHO protocol, with a last follow-up visit at age 3 years were he has reported free of disease.
\end{abstract}

CONCLUSIONS: We describe the largest cases series in Mexico of neonatal leukaemia.

KEYWORDS: neonatal leukaemia; neonatal cancer; acute lymphoid leukaemia; acute myeloid leukaemia; infant; newborn; precursor cell lymphoblastic leukaemia; leukaemia myeloid acute
${ }^{1}$ Médico adscrito a la Unidad de Cuidados Neonatales, Hospital Infantil de México Federico Gómez.

${ }^{2}$ Médico adscrito al Servicio de Oncología, Instituto Nacional de Pediatría.

3jefa de Servicio de Neonatología, Hospital Infantil de México Federico Gómez.

${ }^{4}$ Residente de Oncología Pediátrica, Dirección de Enseñanza, Instituto Nacional de Pediatría.

${ }^{5}$ Médico adscrito al Servicio de Oncología, Hospital Infantil de México Federico Gómez. Jefe del Departamento de Hemato-Oncología del Hospital Infantil de México Federico Gómez.

Correspondence Dra. Marta Zapata-Tarres mzapatatarres@gmail.com

\section{INTRODUCCIÓN}

El cáncer neonatal es aquel grupo de enfermedades malignas que se presentan durante las cuatro primeras semanas de vida extrauterina. Tienen una incidencia de 28/1,000,000 de recién nacidos vivos. ${ }^{1}$ Aunque los tumores neonatales son un grupo de neoplasias paradójicas, representan un gran blanco de atención de los investigadores en el área, ya que presentan un enigma respecto a su etiología, diagnóstico, pronóstico y tratamiento. ${ }^{1-3}$ Las leucemias representan las neoplasias malignas más frecuentes en la edad pediátrica, con una incidencia de 4-5 casos por 100,000 niños; se define como una alteración citogenética que ocasiona una proliferación 
clonal maligna de las células precursoras de linaje linfoide o mieloide, la cual emerge de una célula madre hematopoyética aislada, permitiendo el remplazo de la hematopoyesis normal por un linfoblasto o mieloblasto. De los 7,000 niños diagnosticados por año con cáncer en EE. UU., el $10 \%$ se diagnostica durante el primer año de vida, el $2 \%$ durante el primer mes y solamente el $1 \%$ en el primer día de vida extrauterina. ${ }^{4,5}$

Los casos de leucemias ocurren con menor frecuencia en el periodo perinatal, (menos del $1 \%$ del total de las leucemias) que en la infancia, siendo menos frecuente que otros tumores en esta etapa como el neuroblastoma. Este último se presenta hasta 19 casos por millón de recién nacidos vivos, teniendo la peculiaridad de ser la principal causa de muerte por enfermedad neoplásica en neonatos, así como presentar importantes diferencias en los hallazgos clínicos, factores biológicos, comportamiento y sobre todo respuesta al tratamiento comparada con el mismo diagnóstico en niños mayores. ${ }^{6}$

Hasta el momento no hay estadísticas nacionales. En el Cuadro 1 se muestra la experiencia en 69 años de una sola institución en México, donde las leucemias en la etapa neonatal ocuparon el sexto lugar en frecuencia. ${ }^{7}$ A nivel mundial, la incidencia de leucemias neonatales se reportan en 1 a 5 por cada millón de recién nacidos vivos. ${ }^{8}$ La experiencia en muchos países comprende periodos de 20 a 50 años presentando únicamente decenas de pacientes; siendo estas series de casos las que logran identificar la problemática y estadística de esta neoplasia, que permiten evaluar correctamente las diversas estrategias diagnósticas y terapéuticas empleadas y su impacto en el desenlace.

\section{OBJETIVO}

Describir los casos diagnosticados en el HIMFG debido a su baja frecuencia.
Cuadro 1. Neoplasias malignas en el neonato, experiencia del Hospital Infantil de México Federico Gómez en 69 años ${ }^{7}$

\begin{tabular}{lc|c}
\hline Neoplasia & Frecuencia & $\%$ \\
\hline Neuroblastoma & 18 & 22 \\
$\begin{array}{l}\text { Histiocitosis de células de Lan- } \\
\text { gerhans }\end{array}$ & 12 & 15 \\
Teratoma inmaduro & 10 & 12 \\
\hline Rabdomiosarcoma & 8 & 10 \\
\hline Retinoblastoma & 8 & 10 \\
\hline Leucemias & 5 & 6 \\
\hline Hepatoblastoma & 4 & 5 \\
\hline Otros & 16 & 20 \\
\hline Total & 81 & 100
\end{tabular}

\section{PACIENTES Y MÉTODOS}

Se trata de un estudio retrospectivo, retrolectivo, observacional y descriptivo. Se incluyó a todos pacientes menores de 30 días a los cuales se les realizó el diagnóstico morfológico de leucemias entre el $1^{\circ}$ de enero de 1990 y el $1^{\circ}$ de junio del 2012 en el Hospital. Se excluyeron los casos que no contaran con la información necesaria para documentar el diagnóstico y la evolución. Se revisaron las características clínicas como edad, género, antecedentes perinatales, cuadro clínico al diagnóstico, presencia de visceromegalias, nivel de hemoglobina, leucocitos, blastos, plaquetas, deshidrogenasa láctica y ácido úrico.

Se documentó cual había sido el diagnóstico inicial de ingreso, así como el resultado del perfil para toxoplasmosis, rubeola, citomegalovirus y herpes virus (TORCH). Una vez realizado el diagnóstico de leucemia, se recabó el perfil inmunológico de la misma ya sea por inmunohistoquímica o inmunofenotipo, la presencia de alteraciones citogenéticas de alto riesgo, el cariotipo y el tratamiento recibido. Finalmente se documentó si tuvieron complicaciones y la supervivencia de los pacientes. 
Se consideró un estudio sin riesgo al ser un estudio retrospectivo y se guardó la confidencialidad de los pacientes.

\section{RESULTADOS}

Durante el periodo mencionado se diagnosticaron 3,061 leucemias, de las cuales solo cinco casos $(0.16 \%)$ representaron pacientes diagnosticados en la etapa neonatal. En esta serie reportamos cinco casos de leucemia neonatal, tres del sexo femenino y dos masculinos con edad media al diagnóstico de 21 días de vida, con una edad mínima de 17 días y máxima de 26 días.

La leucemia mieloide aguda fue más frecuente con tres casos. En ninguno de los casos se reportó alguna translocación o alteración citogenética de alto riesgo. En el Cuadro 2 se muestran las características clínicas, de laboratorio, la clasificación de la leucemia, el tratamiento, la supervivencia y las complicaciones de estos cinco pacientes reportados.

\section{DISCUSIÓN}

Analizando los resultados, tres de los cinco casos $(60 \%)$ fueron leucemias mieloides y dos pacientes linfoides. Esto concuerda con la literatura internacional que reporta leucemias mieloides de un 56 a 64\% y linfoides de un 21 a 38\%. . $^{8-11}$

Dentro de las leucemias mieloides se encontró un caso de leucemia mieloide aguda con maduración mínima (M1, por clasificación del grupo cooperativo French-American-British, FAB por sus siglas en inglés), un caso con megacarioblástica (M7) y un caso con maduración (M2). En la literatura internacional ${ }^{8-12}$ las leucemias mieloides son monoblásticas (M5) hasta en un $50 \%$, sin encontrar pacientes con este diagnóstico en nuestra serie. Como se ha descrito nuestro paciente con síndrome Down cursó con
LMA M7. Este tipo de morfología se ha descrito también en los síndromes mieloproliferativos transitorios, diagnóstico diferencial de importancia en pacientes con síndrome de Down en la etapa neonatal. ${ }^{12}$

No se identificaron factores de riesgo ambientales o genéticos buscados intencionalmente en esta serie de casos. Los factores de riesgo descritos para leucemias en menores de un año, como son uso materno de mariguana, alcohol y dieta rica en flavonoides, no se encuentran asociados en nuestros pacientes. ${ }^{13,14}$ Los únicos datos presentes que se describieron fueron el antecedente de exposición de los padres a pesticidas en el paciente 5; sin embargo, este factor de riesgo reportado para tumores en la etapa neonatal no se ha descrito para leucemia neonatal. Se reporta tabaquismo en los padres de los pacientes dos y cinco; sin embargo, no existe relación estrecha reportada para leucemia neonatal. No contamos con estudios genéticos dentro del abordaje de los cinco casos para establecer la participación del gen MLL y sus rearreglos, como factor de riesgo genético, el cual se encuentra bien descrito en los casos de pacientes tanto en la etapa neonatal así como en lactantes. ${ }^{15}$

A pesar de que todos nuestros pacientes se presentaron con hepatomegalia y/o esplenomegalia como datos clínicos al diagnóstico de ingreso ninguno tuvo sospecha prenatal de leucemia aguda. La presencia de hepatoesplenomegalia, hydrops fetal y/o polihidramnios son datos que apoyan a establecer sospecha diagnóstica de leucemia antenatal; puede corroborarse el diagnóstico por medio de fetoscopia y muestreo de sangre umbilical con tinción Wright-Giemsa. Al igual que los hallazgos por ultrasonografía estructural, la presencia de anemia fetal puede representar un dato de sospecha para leucemia neonatal, la cual puede diagnosticarse y vigilarse mediante Doppler de la arteria cerebral media de manera previa al nacimiento, encontrando inclusive 
Cuadro 2. Características de los pacientes con leucemia neonatal (continúa en la siguiente página)

\begin{tabular}{|c|c|c|c|c|c|}
\hline Paciente & 1 & 2 & 3 & 4 & 5 \\
\hline $\begin{array}{l}\text { Edad al } \\
\text { diagnóstico }\end{array}$ & 24 días & 17 días & 26 días & 21 días & 19 días \\
\hline Género & Femenino & Masculino & Femenino & Femenino & Masculino \\
\hline $\begin{array}{l}\text { Antecedente } \\
\text { perinatal }\end{array}$ & $\begin{array}{l}\text { Gesta } 2 \\
\text { Peso } 2550 \mathrm{~g}\end{array}$ & $\begin{array}{l}\text { Gesta } 2 \\
\text { Peso } 2400 \mathrm{~g} \\
\text { Tabaquismo y } \\
\text { alcoholismo en el } \\
\text { padre }\end{array}$ & $\begin{array}{l}\text { Gesta } 2 \\
\text { Peso } 2050 \mathrm{~g}\end{array}$ & $\begin{array}{l}\text { Gesta } 3 \\
\text { Peso } 3260 \mathrm{~g}\end{array}$ & $\begin{array}{l}\text { Gesta } 3 \\
\text { Peso } 3500 \mathrm{~g} \\
\text { Tabaquismo en } \\
\text { el padre. Contac- } \\
\text { to ocasional con } \\
\text { pesticidas en el } \\
\text { padre }\end{array}$ \\
\hline Clínica inicial & $\begin{array}{l}\text { Fiebre, vómito y } \\
\text { cianosis }\end{array}$ & $\begin{array}{l}\text { Fiebre, leucemia } \\
\text { cutis, síndrome } \\
\text { anémico }\end{array}$ & $\begin{array}{l}\text { Fiebre, leucemia } \\
\text { cutis, síndrome } \\
\text { hemorragíparo }\end{array}$ & $\begin{array}{l}\text { Fiebre, epífora } \\
\text { y aumento del } \\
\text { volumen de la he- } \\
\text { micara izquierda }\end{array}$ & $\begin{array}{l}\text { Leucemia cutis y } \\
\text { biometría hemá- } \\
\text { tica anormal }\end{array}$ \\
\hline $\begin{array}{l}\text { Presencia de } \\
\text { visceromegalias }\end{array}$ & Sí & Sí & Sí & Sí & Sí \\
\hline $\begin{array}{l}\text { Hemoglobina (g/ } \\
\text { dL) }\end{array}$ & 9.4 & 9.2 & 8.6 & 8.2 & 13 \\
\hline Leucocitos/mL & 292,000 & 201,500 & 196,000 & 175,000 & 241,000 \\
\hline Blastos SP & $91 \%$ & $76 \%$ & $82 \%$ & $98 \%$ & $77 \%$ \\
\hline Plaquetas /mL & 199,000 & 43,000 & 82,000 & 21,000 & 92,000 \\
\hline DHL (U/L) & 1,143 & 333 & 756 & 1,274 & 1,499 \\
\hline $\begin{array}{l}\text { Acido úrico } \\
\text { (mg/dL) }\end{array}$ & 6.7 & 5.6 & 7.2 & 13.5 & 8 \\
\hline $\begin{array}{l}\text { Diagnóstico } \\
\text { al ingreso }\end{array}$ & $\begin{array}{l}\text { Sepsis neonatal } \\
\text { Hiperlecucocitosis } \\
\text { Hepatoesplenomegalia } \\
\text { Hiperuricemia } \\
\text { Anemia }\end{array}$ & $\begin{array}{l}\text { Sepsis neonatal } \\
\text { Hiperleucocitosis } \\
\text { Hepatomegalia } \\
\text { Hiperuricemia } \\
\text { Anemia } \\
\text { Síndrome coles- } \\
\text { tásico }\end{array}$ & $\begin{array}{l}\text { Sepsis } \\
\text { neonatal } \\
\text { Hiperleucocitosis } \\
\text { Hepatomegalia } \\
\text { Hiperuricemia } \\
\text { Anemia } \\
\text { Síndrome de } \\
\text { Down } \\
\text { Cardiopatía } \\
\text { congénita } \\
\text { Enfermedad } \\
\text { hemorrágica } \\
\text { del RN }\end{array}$ & $\begin{array}{l}\text { Hiperleucocitosis } \\
\text { Hepatoespleno- } \\
\text { megalia } \\
\text { Hiperuricemia } \\
\text { Anemia } \\
\text { Pb. neoplasia }\end{array}$ & $\begin{array}{l}\text { Pb. leucemia } \\
\text { neonatal } \\
\text { Hepatoespleno- } \\
\text { megalia } \\
\text { Hiperuricemia } \\
\text { Síndrome de lisis } \\
\text { tumoral }\end{array}$ \\
\hline TORCH & Negativo & Negativo & Negativo & Negativo & Negativo \\
\hline $\begin{array}{l}\text { Diagnóstico } \\
\text { oncológico }\end{array}$ & LLA L2* & LMA M1* & LMA M7* & LLA L1* & LMA M2* \\
\hline $\begin{array}{l}\text { Inmunohistoquí- } \\
\text { mica }\end{array}$ & Linfoide & Mieloide & Mieloide & Linfoide & Mieloide \\
\hline $\begin{array}{l}\text { Citogenética para } \\
\text { traslocaciones de } \\
\text { alto riesgo }\end{array}$ & Negativa & Negativa & Negativa & Negativa & Negativa \\
\hline Cariotipo & $46 X X$ & $46 X Y$ & $46 X X$ & $46 X X$ & $46 X Y$ \\
\hline
\end{tabular}


Cuadro 2. Características de los pacientes con leucemia neonatal (continuación)

\begin{tabular}{|c|c|c|c|c|c|}
\hline Paciente & 1 & 2 & 3 & 4 & 5 \\
\hline Tratamiento & Esteroide & Esteroide & $\begin{array}{l}\text { Esteroide e } \\
\text { inducción }\end{array}$ & $\begin{array}{l}\text { Esteroide e induc- } \\
\text { ción }\end{array}$ & $\begin{array}{l}\text { Exanguinotrans- } \\
\text { fusión. } \\
6 \text { ciclos de } \\
\text { ATEDox }\end{array}$ \\
\hline Complicaciones & $\begin{array}{l}\text { Sepsis, trombocitope- } \\
\text { nia, anemia, } \\
\text { neutropenia y fiebre }\end{array}$ & $\begin{array}{l}\text { Sepsis, } \\
\text { trombocitopenia, } \\
\text { anemia, } \mathrm{n} \\
\text { eutropenia (colitis } \\
\text { neutropénica) } \\
\text { y fiebre, }\end{array}$ & $\begin{array}{l}\text { Anemia, se ma- } \\
\text { neja infección } \\
\text { por VSR y se } \\
\text { traslada a su } \\
\text { lugar de origen } \\
\text { por máximo } \\
\text { beneficio }\end{array}$ & $\begin{array}{l}\text { Insuficiencia } \\
\text { respiratoria, } \\
\text { trombocitopenia, } \\
\text { anemia, neutro- } \\
\text { penia y fiebre }\end{array}$ & $\begin{array}{l}\text { Insuficiencia } \\
\text { renal aguda. } \\
\text { Sépsis. } \\
\text { Hiperuricemia } \\
\text { Neutropenia y } \\
\text { fiebre }\end{array}$ \\
\hline Supervivencia & 57 días & 89 días & 78 días & 176 días & $\begin{array}{l}\text { Vivo en se- } \\
\text { guimiento por } \\
\text { Oncología y } \\
\text { Neonatología a } \\
3 \text { años }\end{array}$ \\
\hline AMO al Dx & Día 3 de Ingreso & Día 2 de Ingreso & $\begin{array}{l}\text { Día } 10 \text { de } \\
\text { Ingreso }\end{array}$ & Día 1 de Ingreso & Día 1 de ingreso \\
\hline AMO remisión & Día 30 de Ingreso & Día 32 de Ingreso & No & No & $\begin{array}{l}\text { Negativo al } 27 \\
\text { sept. } 2010\end{array}$ \\
\hline
\end{tabular}

Dx: diagnóstico; DHL: deshidrogenasa láctica; Hb: hemoglobina; SP: sangre periférica; Ac: ácido; TORCH: toxoplasma, rubeola, citomegalovirus, herpes y otros; AMO: aspirado de médula ósea; VSR: virus sincicial respiratorio; LLA: leucemia linfoblástica aguda; LMA: leucemia mieloblástica aguda; Pb: probable; RN: recién nacido; ATEDox: esquema de quimioterapia citarabina, etopósido, 6 Mercaptopurina y Doxorrubicina. *Sistema FAB: clasificación morfológica por la sociedad Franco-Americano-Británico.

reportes de fetos con síndrome de Down con diagnóstico prenatal de leucemia aguda desde la semana 33 de gestación por cordoscentesis. ${ }^{16}$

Dentro de la clínica para el diagnóstico se encontró que todos nuestros casos presentaron hepatoesplenomegalia, un dato clínico reportado hasta en un $80 \%$ en otras series de casos. ${ }^{8-12}$ El diagnóstico de leucemia cutis se realizó en tres pacientes $(60 \%)$, comparado con el $7.7 \%$ (cuatro pacientes de 52) reportado por Isaacs. ${ }^{9}$ Todos nuestros pacientes, tanto de leucemias mieloides como linfoides, presentaron hiperleucocitosis al diagnóstico, descrito hasta en un $85 \%$ de las leucemias linfoides y $49 \%$ de las leucemias mieloides. ${ }^{8-12}$
En todos los pacientes se realizó diagnóstico diferencial para infecciones por el complejo $\mathrm{TORCH}$, incompatibilidad a grupo y otros tipos de cánceres como neuroblastoma e histiocitosis de células de Langerhans. En el Cuadro 3 se muestran los diagnósticos diferenciales de la leucemia neonatal más frecuentes.

Cuatro pacientes recibieron manejo para lisis tumoral con alopurinol, medicamento base del tratamiento de esta complicación metabólica; sin embargo, no descrito de manera sistemática en la etapa neonatal. Solo un paciente (caso cinco) completó el ciclo para leucemias mieloides con citarabina (Ara-C), 6- mercaptopurina (6-MP), etopósido (VP-16), doxorrubicina y metotrexate 
Cuadro 3. Diagnóstico diferencial de leucemia neonatal

Enfermedades infecciosas: TORCH e Infecciones bacterianas (Listeria monocytogenes).

Enfermedades hematológicas. Procesos Inmunes (Incompatibilidad Rh, $\mathrm{ABO}^{*}$ o subgrupos). Procesos no inmunes (talasemias, infecciones intrauterinas por parvovirus, anemia congénita, anemia de Diamond-Blackfan, hipoxia intrauterina o transfusión gemelo-gemelo).

Otras neoplasias. Neuroblastoma.

Histiocitosis. Histiocitosis de células de Langerhans, linfohistiocitosis hemafagocítica, xantugranuloma juvenil.

*Sistema de clasificación de grupo sanguíneo (ABO).

(ATEDoxNOPHO al 50\%) con remisión documentada por aspirado de médula ósea. Durante su seguimiento por consulta externa de oncología se reportó un nuevo aspirado de médula ósea en remisión posterior al cual los padres rechazaron el tratamiento de mantenimiento y se pierde su seguimiento. Regresa a la edad de tres años refiriéndose biometrías hemáticas normales hasta ese momento. Existen casos reportados en todas las edades de pacientes que después de entrar en remisión pueden tener supervivencias prolongadas.

Con estos datos obtenemos una supervivencia global del $20 \%$, y del $33 \%$ al incluir únicamente leucemias mieloides agudas. En la literatura internacional se describe una supervivencia para las linfoides de menos del $10 \%$, y para las mieloides en un 25\%. ${ }^{8-12}$ Es difícil comparar la información debido a que se trata de una serie de casos.

En México existen dos publicaciones reportando leucemias neonatales. Rendón-García y colaboradores ${ }^{17}$ describen dos casos en 27 años en el Hospital Infantil de Sonora, una LMA M4 con Síndrome de Down y una LMA indiferenciada. Por su parte, Miranda del Olmo y colaboradores $^{18}$ reportan una leucemia linfoblástica aguda L2 en un paciente con síndrome de Down. No se reporta supervivencia de ninguno de los pacientes. En el Cuadro 4 se resumen los casos reportados de leucemias en el neonato en México. Esta información nos muestra la dificultad del diagnóstico de esta patología. Estos datos podrían permitir que en este grupo etario donde los síntomas son tan inespecíficos se aumente la sospecha diagnóstica de leucemia y mejore su pronóstico a mediano plazo.

\section{CONCLUSIÓN}

La leucemia neonatal es un padecimiento poco frecuente, con un pronóstico sombrío, el cual puede mejorar con el diagnóstico oportuno. El impacto en el desenlace de estos pacientes se logrará al poder considerar la sospecha diagnóstica y confirmación prenatal, así como la instauración de un manejo agresivo con qui-

Cuadro 4. Leucemias neonatales reportadas en México

\begin{tabular}{|c|c|c|c|c|c|}
\hline & Mieloide & Morfología & Linfoide & Morfología & Supervivencia \\
\hline Miranda y col. 2002 & - & - & 1 paciente & LLA L2* & $0 \%$ \\
\hline Rendón y col. 2006 & 3 pacientes & $\begin{array}{c}\text { LMA M4* } \\
\text { LMA M4* } \\
\text { LMA Indiferenciada }\end{array}$ & - & - & $0 \%$ \\
\hline Zapata y col. 2016 & 3 pacientes & $\begin{array}{l}\text { LMA M1* } \\
\text { LMA M2* } \\
\text { LMA M7* }\end{array}$ & 2 pacientes & $\begin{array}{l}\mathrm{LLA} \text { L1* } \\
\mathrm{LLA} \text { L2* }\end{array}$ & 1 paciente $(20 \%)$ \\
\hline
\end{tabular}

*Clasificación morfológica por la sociedad Franco-Americano-Británico (FAB). 
mioterapia de forma temprana, lo que traduce la importancia del manejo con quimioterapia combinada pero además la terapia de soporte (hemoderivados, antimicrobianos, antifúngicos, nutricional, etc.) mejorando las posibilidades de supervivencia.

Ante el caso de un paciente con sospecha de leucemia aguda neonatal, siempre se deben tener presentes diagnósticos diferenciales, sobre todo del tipo infeccioso y hematológico, sin retrasar el abordaje diagnóstico de leucemia.

Esta es la serie más grande de leucemias neonatales reportada en México y la única donde se logró la administración completa del tratamiento con quimioterapia, que permitió la supervivencia de un paciente. Se trata de una serie de casos lo cual conlleva deficiencias metodológicas importantes, pero es el antecedente para proponer un ensayo clínico nacional dada la baja frecuencia de la enfermedad.

\section{REFERENCIAS}

1. Vormoor J. Editorial: Leukaemia and cancer in neonates. Semin Fetal Neonatal Med. 2012;17:183-4.

2. Isaacs H Jr. Tumors of the fetus and newborn. Vol. 35 . Philadelphia: WB Saunders; 1997. p. 278-97.

3. Berry PJ. Congenital tumours. Fetal and Neonatal Pathology. 2nd ed. Berlin: Springer-Verlag; 1993. p. 273-91.

4. Isaacs $\mathrm{H}$ Jr. Congenital and neonatal malignant tumors. A 28 years experience at Children's Hospital of Los Angeles. Pediatr Hematol Oncol. 1987;9:121-9.

5. Bader JL, Miller RW. US cancer incidence and mortality in the first year of life. Am J Dis Child. 1979;133:157-9.

6. Vasilatou-Kosmidis H. Cancer in neonates and infants. Med Pediatr Oncol. 2003;41:7-9.
7. Cicero Oneto C, Zapata Tarrés M, Flores Toscano K, Flores Montes O. Tumores sólidos malignos neonatales en el Hospital Infantil de México Federico Gómez. Experiencia de 69 años. GAMO. 2013;12:143-9.

8. Johnson K, Roesler M, Linabery A, Hilden J, Davies S, Ross J. Infant leukemia and congenital abnormalities: A Children's Oncology Group study. Pediatric Blood Cancer. 2010;55:95-9.

9. Isaacs H Jr. Fetal and neonatal leukemia. J Pediatr Hematol Oncol. 2003;25:348-1.

10. Bresters D, Reus A, Veerman A, Van Wering E, Van Der Does-van den Berg A, Kaspers G. Congenital Leukaemia: the Dutch experience and review of the literature. $\mathrm{Br} J$ Haematol. 2002;117:513-24.

11. Ishii E, Oda M, Kinugawa N, Oda T, Takimoto T, Suzuki N, et al. Features and outcome of neonatal leukemia in Japan: Experience of the Japan Infant Leukemia Study Group. Pediatr Blood Cancer. 2006;47:268-72.

12. Brink DS. Transient leukemia (transient myeloproliferative disorder, transient abnormal myelopoiesis) of Down syndrome. Adv Anat Pathol. 2006;13:256-62.

13. Sgu XO, Ross JA, Pendergrass T, Reaman G, Lampkin B, Robinson L. Parental alcohol consumption, cigarette smoking, and risk of infant leukemia: a Childrens Cancer Group Study. J Natl Cancer Inst. 1996;88:24-31.

14. Robinson LL, Buckley JD, Daigle AE et al. Maternal drug use and risk of childhooldnonlumphoblasticleukemia among offspring. An epidemiologic investigation implicating marijuana (a report from the Children's Cancer Study Group). Cancer. 1989;63:1904-11.

15. Hess JL, Yu BD, Li B et al. Defects in yolk sac hematopoiesis in M11-null embryos. Blood. 1997;90:1799-1806.

16. Foucar K, Friedman K, Llewellyn A et al. Prenatal diagnosis of myeloproliferative disorder via percutaneous umbilical blood sampling. Report of two cases in fetuses affected by Down's syndrome. Am J Pathol. 1992;97:584-90.

17. Rendón García H, Covarrubias Espinoza G, Ornelas Sánchez M, Rivera Gómez R. Leucemia del Recién Nacido. Bol Clin Hosp Infant Edo Son. 2006;23:98-101.

18. Miranda H, Cardiel L, Verdusco M, Durán MA, Bolea V, Reynoso E et al. Un caso de leucemia congénita y revisón del tema. Rev Mex Pediatr. 2002;69;201-5. 